\title{
AN ADAPTIVE FINITE ELEMENT METHOD FOR SOLVING A DOUBLE WELL PROBLEM DESCRIBING CRYSTALLINE MICROSTRUCTURE
}

\author{
ANDREAS PROHL ${ }^{1}$
}

\begin{abstract}
The minimization of nonconvex functionals naturally arises in materials sciences where deformation gradients in certain alloys exhibit microstructures. For example, minimizing sequences of the nonconvex Ericksen-James energy can be associated with deformations in martensitic materials that are observed in experiments [2,3]. - From the numerical point of view, classical conforming and nonconforming finite element discretizations have been observed to give minimizers with their quality being highly dependent on the underlying triangulation, see [8,24,26,27] for a survey. Recently, a new approach has been proposed and analyzed in $[15,16]$ that is based on discontinuous finite elements to reduce the pollution effect of a general triangulation on the computed minimizer. The goal of the present paper is to propose and analyze an adaptive method, giving a more accurate resolution of laminated microstructure on arbitrary grids.
\end{abstract}

AMS Subject Classification. 65K10, 65M50, 65N30, 73C50, 73S10.

Received: February 27, 1998. Revised: September 12, 1998.

\section{INTRODUCTION}

In modern materials sciences, alloys are the subject of research that exhibit a memory shape effect: when cooled beyond a certain critical temperature, the crystal structure changes rapidly, and a new configuration of the atoms with less symmetry properties (martensitic phase) can be observed, exhibiting microstructure. When heated, the original (austenite) phase in the alloy is taken again and no microstructure prevails any more. - A mathematical description that is based on the elastic energy minimization has been developed for the equilibria of certain martensitic crystals in $[2,3,12-14]$. The invariance of the energy density with respect to symmetry-related states of the material and their rotational invariance are the reasons for the nonconvexity of the density and multiple energy wells. We e.g. refer to [27] for a survey of different phase transformations that can be observed in experiments and can be described by this model. Mathematically, for a large class of boundary conditions, the gradients of energy-minimizing sequences of deformations must oscillate between the energy wells to allow the energy to converge to the lowest possible value. It is because of the non-quasiconvex character of the energy that the weak limit of these minimizing sequences is no minimizer of the problem, in general.

Keywords and phrases. Adaptive algorithm, finite element method, nonconvex minimization, multi-well problem, microstructure, multiscale, nonlinear elasticity, shape-memory alloy, materials science.

${ }^{1}$ Mathematisches Seminar, Christian-Albrechts-Universität Kiel, Ludewig-Meyn-Str. 4, 24098 Kiel, Germany.

e-mail: apr@numerik.uni-kiel.de

(C) EDP Sciences, SMAI 1999 
TABLE 1. Summary of convergence results for the energy and other crucial quantities for different finite element methods. See the text for an explanation of the employed notation.

\begin{tabular}{|l|c|c|c|c|}
\hline Finite element method & $\mathcal{E}_{h}\left(u_{h}\right)$ & $\left\|u_{h}-F_{\lambda} x\right\|$ & $\left\|\left(\nabla u_{h}-F_{\lambda}\right) w\right\|$ & $\left|\frac{\mu\left(\omega_{\rho}^{\ell}\left(u_{h}\right)\right)}{\mu(\omega)}-\lambda^{\ell}\right|$ \\
\hline $\begin{array}{l}\text { classical conforming and } \\
\text { nonconforming methods, } \\
\text { see }[23,26]\end{array}$ & $\mathcal{O}\left(h^{1 / 2}\right)$ & $\mathcal{O}\left(h^{1 / 8}\right)$ & $\mathcal{O}\left(h^{1 / 8}\right)$ & $\mathcal{O}\left(h^{1 / 16}\right)$ \\
\hline $\begin{array}{l}\text { discontinuous method, } \\
\text { see }[15]\end{array}$ & $\mathcal{O}\left(h^{2}\right)$ & $\mathcal{O}\left(h^{1 / 4}\right)$ & $\mathcal{O}\left(h^{1 / 4}\right)$ & $\mathcal{O}\left(h^{1 / 8}\right)$ \\
\hline $\begin{array}{l}\text { discontinuous, adaptive } \\
\text { method }\end{array}$ & $\mathcal{O}\left(h^{2}\right)$ & $\mathcal{O}\left(h^{3 / 10}\right)$ & $\mathcal{O}\left(h^{3 / 10}\right)$ & $\mathcal{O}\left(h^{3 / 20}\right)$ \\
\hline
\end{tabular}

From the numerical point of view, standard finite element methods [of conforming or nonconforming type (i.e., Crouzeix or rotated (bi-, tri-)linear finite elements)] turn out to be capable of simulating deformations giving microstructure, reliably provided triangulations are used that are aligned to the microstructure, see $[10,17,24,26,27]$. In order to free the numerical scheme from these restrictions and to make a finite element model more flexible for instance for problems with acting forces and/or complex changing (in time) microstructures, a new ansatz based on discontinuous elements was recently proposed and analyzed in $[15,16]$. The improved performance of this method is tested in computational experiments and is supported through a rigorous convergence analysis, giving drastically improved orders of convergence, if compared to results for classical conforming and nonconforming ansatzes. We refer to Table 1 for a comparison of the different methods.

The goal of the present paper is to propose a new adaptive method in order to resolve laminated microstructure, with the main focus on the verification of improved convergence statements for it. At first glance, adaptivity does not seem to make any sense in the context of microstructures under consideration, since there is no scaling in the solution where an adaptivity strategy can be based on. On the other hand, since we are using discontinuous ansatz functions, an adaptivity criterion can be based on the degree of discontinuity of a computed solution, i.e., on the height of inter-element jumps. We will outline the strategy in Section 3, and propose the new adaptive algorithm there. - The application of such an adaptivity strategy allows for convergence results that are superior to those of previous methods collected in Table 1, and also for the method that is based on discontinuous ansatz functions. We refer to Theorem 3.1 in Section 3 for further details on the performance of this algorithm.

The remainder of the article is organized as follows: Section 2 provides the reader with the mathematical setting of the problem that we consider. The adaptive algorithm as well as the main results of convergence are presented in Section 3. Their verification is subject of Section 4.

\section{THE NONCONVEX ENERGY FUNCTIONAL}

A common example of microstructures is given by simple laminates in which the deformation gradient oscillates on an infinitesimal scale in alternating bands between two states that correspond to two symmetry-related variants of the martensitic phase. It follows from the frame-indifference principle that the minimum value of the elastic energy density is attained on multiple, rotationally invariant wells. We study in this paper the approximation of deformations of martensitic crystals which can undergo an orthorhombic to monoclinic transformation, giving rise to a double well potential. We claim that the analysis can easily be extended to more complicated phase transitions like, e.g., the "triple well case" describing cubic to tetragonal phase transitions in atomic lattice structure; we refer to [24] for a corresponding study of the rotated (bi-, tri-)linear finite elements. 
The energy functional that will be investigated in the following is of the form

$$
\mathcal{E}(v)=\int_{\Omega} \phi(\nabla v(x)) \mathrm{d} \Omega
$$

for $\phi(\cdot)$ the nonconvex energy density and admissible deformations $v \in \mathcal{A}$ to be defined below. For example, the following conditions on $\phi(\cdot)$ are satisfied by the physically meaningful Ericksen-James energy, see e.g. $[2,3,12-14,27]$. - Let us suppose that each well is of the form $\mathcal{U}_{\ell}=S O(3) U_{\ell}$, with $S O(3)$ being the group of proper rotations and the $U_{\ell}, \ell=1,2$ representing martensitic variants. In the double well case, we shall assume (see [27]) that these wells are rank-one connected, i.e., there are $F_{\ell} \in \mathcal{U}_{\ell}, \ell=1,2$, such that the Hadamard condition is satisfied. This means that there are two non-vanishing vectors $a \in \mathbb{R}^{3}$ and $n \in \mathbb{R}^{3}$ such that

$$
F_{2}=F_{1}+a \otimes n
$$

Without loss of generality, we assume $|n|=1$.

We continue with the presentation of the general setting: $\Omega \subset \mathbb{R}^{3}$ is the reference domain of the crystal, which is assumed to be polygonal. Furthermore, the mapping $u: \Omega \rightarrow \mathbb{R}^{3}$ represents a continuous deformation of the crystal, with corresponding deformation gradients $\nabla u: \Omega \rightarrow \mathbb{R}^{3 \times 3}$. - The energy density is assumed to satisfy

$$
\begin{aligned}
& \phi(A) \geq 0, \quad \forall A \in \mathbb{R}^{3 \times 3}, \\
& \phi(A)=0 \quad \Longleftrightarrow A \in \mathcal{U}=\mathcal{U}_{1} \cup \mathcal{U}_{2} .
\end{aligned}
$$

We shall also assume that the energy density $\phi$ grows quadratically from the energy wells, that is,

$$
\phi(F) \geq \kappa\left|\|F-\pi(F) \mid\|^{2}, \quad \forall F \in \mathbb{R}^{3 \times 3},\right.
$$

with $\kappa>0$ a constant and $\pi: \mathbb{R}^{3 \times 3} \rightarrow \mathcal{U}$ a Borel measurable projection that is defined by

$$
\left\||| F-\pi(F)||\left|=\min _{G \in \mathcal{U}}\right|\right\| F-G \mid \|, \quad \forall F \in \mathbb{R}^{3 \times 3} .
$$

Here, ||$|\cdot|||$ denotes the Frobenius norm, i.e., ||$|A|||=\left(\sum_{i, j=1}^{3} A_{i j}^{2}\right)^{1 / 2}$. Note that the projection $\pi(F)$ exists for any $F \in \mathbb{R}^{3 \times 3}$ since $\mathcal{U}$ is compact, although it may not be unique.

As already mentioned, we are interested in the approximation of a unique simple laminated microstructure. This can be enforced through the prescription of compatible affine boundary conditions,

$$
u(x)=F_{\lambda} x, \quad \forall x \in \partial \Omega,
$$

where

$$
F_{\lambda}=\lambda F_{1}+(1-\lambda) F_{2},
$$

and $\lambda \in[0,1]$ represents the mass fraction of the two variants. - The problem can then be stated in the following way:

$$
\inf \{\mathcal{E}(v): v \in \mathcal{A}\}
$$

and

$$
\mathcal{A}=\left\{v \in W^{1, \infty}\left(\Omega ; \mathbb{R}^{3}\right):\left.v(x)\right|_{\partial \Omega}=F_{\lambda} x\right\}
$$


Throughout the remainder of the paper, we make use of the following standard notation, see [9]. For any integer $k \geq 0$ and $p \in[1, \infty]$, we define the space

$$
W_{h}^{k, p}(\Omega) \equiv\left\{v \in L^{p}(\Omega):\left.v\right|_{K} \in W^{k, p}(K), \forall K \in \mathcal{T}_{h}\right\}
$$

and we equip $W_{h}^{k, p}(\Omega)$ with the standard norms $|\cdot|_{k, p} \equiv\left(\sum_{K \in \mathcal{T}_{h}}|\cdot|_{k, p, K}^{p}\right)^{1 / p}$, for $1 \leq p<\infty$ and $\max _{K \in \mathcal{T}_{h}} \mid \cdot$ $\left.\right|_{k, \infty, K}$, for $p=\infty$. Correspondingly, $\|\cdot\|_{k, p}$ is defined, using norms instead of semi-norms. Subsequently, we will omit the indices in situations where the meaning of the notation is clear from the context.

\section{Proposal of the Algorithm}

We already mentioned the fact that classical conforming or nonconforming discretization ansatzes for the minimization of the energy function $\mathcal{E}(\cdot)$ (or its modification $\mathcal{E}_{h}(\cdot)$ for the nonconforming approach) give minimizers with their quality being highly mesh dependent. This is because of too strict continuity constraints of the finite element functions polluting the solution on meshes that are not aligned to the laminated microstructure. In order to circumvent this problem and to have a method that is essentially independent on the choice of the present triangulation, a finite element approach based on discontinuous ansatzes has been proposed in [15]. Here, the inter-element continuity constraint of the computed deformation is taken into account through a penalty term that is added to the bulk energy functional and which takes into account large jumps across inter-element faces. In the following, we use the notation $\left.\left[v_{h}\right]\right|_{\Gamma^{\prime}}:=\left.v_{h}\right|_{K^{+}}-\left.v_{h}\right|_{K^{-}}$, for a fixed but arbitrary orientation of the mesh and any two adjacent elements $K^{+}, K^{-}$, such that $\partial K \supset \Gamma=\overline{K^{+}} \cap \overline{K^{-}}$. Further, an analogous idea relaxes the boundary values in a way that small deviations from the prescribed boundary data are permitted. As a consequence, this reduces the pollution of the computed deformation through averaged boundary values on general meshes. Then, the energy functional of the numerical model is as follows.

We are given a triangulation $\mathcal{T}_{h}$ of the domain $\Omega$ that can be parametrized by $h>0$. Consider element-wise linear deformations $v_{h} \in \mathcal{A}_{h} \equiv \prod_{K \in \mathcal{T}_{h}} \mathcal{P}_{1}(K)$, having an energy

$$
\begin{aligned}
\mathcal{E}_{h}^{\beta}\left(v_{h}\right)= & \sum_{K \in \mathcal{T}_{h}} \int_{K} \phi\left(\nabla v_{h}(x)\right) \mathrm{d} \Omega+\alpha_{11}\left(\sum_{K \in \mathcal{T}_{h}} h^{1-\beta} \int_{\partial K}\left|\left[v_{h}\right](x)\right| \mathrm{d} \sigma\right)^{2} \\
& +\alpha_{12}\left(\sum_{K \in \mathcal{T}_{h}} h^{1-\beta} \int_{\partial K}\left|\left[v_{h}\right](x)\right|^{2} \mathrm{~d} \sigma\right)+\alpha_{2} \sum_{K \in \mathcal{T}_{h}} h^{2 \beta} \int_{\partial K \cap \partial \Omega}\left|v_{h}(x)-F_{\lambda} x\right|^{2} \mathrm{~d} \sigma,
\end{aligned}
$$

and do the following minimization, for a given parameter $\beta \in[0,1]$,

$$
\min _{v_{h} \in \mathcal{A}_{h}} \mathcal{E}_{h}^{\beta}\left(v_{h}\right) .
$$

In this algorithm, the parameter $\beta$ plays a role to distinguish between the basic discretization scale $\mathcal{O}(h)$ and a scale for laminates $\mathcal{O}\left(h^{1-\beta}\right)$, i.e., the structure of the solution. As far as the latter statement is concerned, we mean that deformations $v_{h} \in \mathcal{A}_{h}$ are energetically preferred that show laminated microstructure with the (averaged) band width of size $\mathcal{O}\left(h^{1-\beta}\right)$. As it turns out from the analysis in [15], the algorithm gives most accurate computations of the microstructure for the choice $\beta=1 / 2$. The $\alpha_{11}, \alpha_{12}, \alpha_{2}$ are positive numbers of order $\mathcal{O}(1)$ each to relatively balance the physical and different numerical effects in the algorithm.

Based on the idea of distinguishing between the scaling of the laminated microstructure and transitions between related variants, which has proved to be fruitful in the formulation of the previous algorithm, we will now extend this idea to the context of adaptivity. The idea is now to expand the area "supporting" variants by reducing the area where variant transitions occur at the same time.

The adaptive algorithm proposed here consists of two parts: a refinement criterion of the present underlying triangulation, and the formulation of an energy functional $\mathcal{E}_{h, \beta}^{\beta_{i}}(\cdot)$ that changes for each new triangulation $\mathcal{T}_{h}^{i}$, 

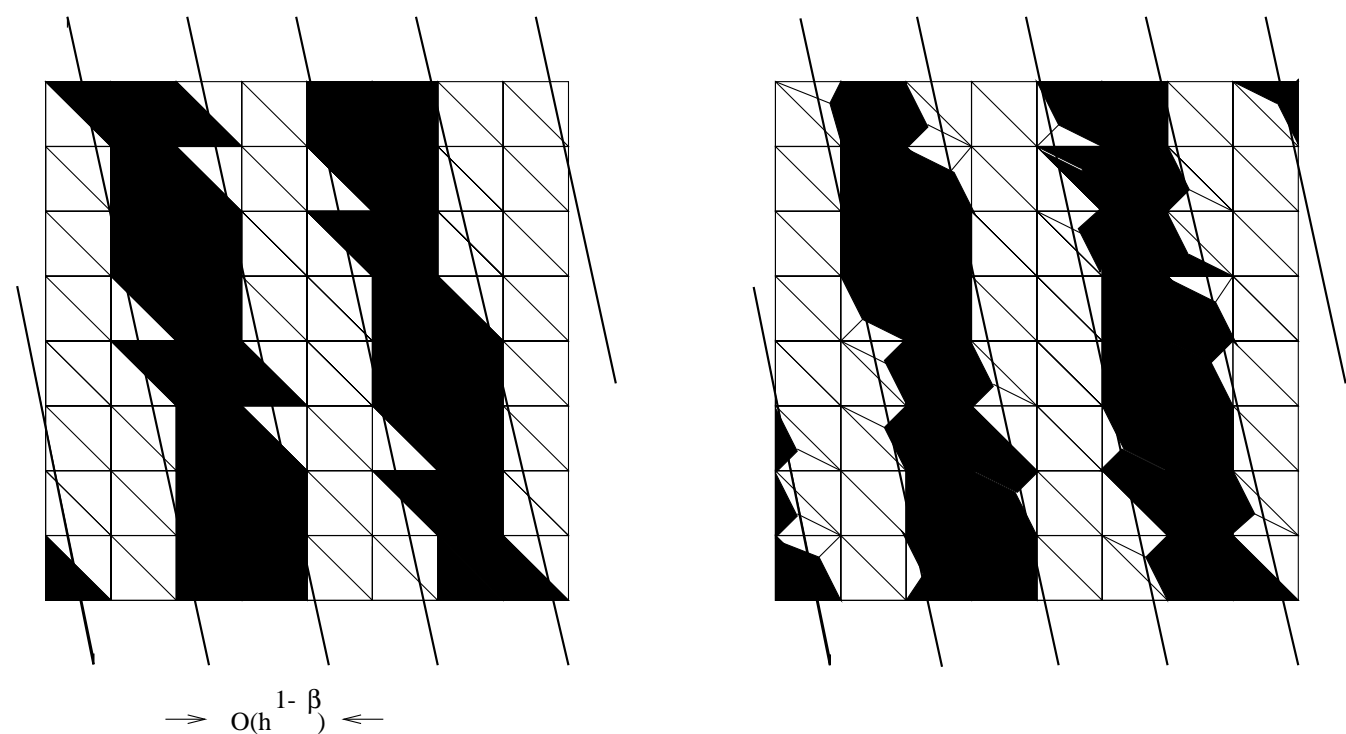

FIGURE 1. Sketch of the adaptive refinement strategy in 2D: white corresponds to $\nabla u=F_{1}$, black to $\nabla u=F_{2}$. The figures show a possible first adaptive refinement of a given basic triangulation.

parametrized by $i \geq 0$, by using different values $\beta_{i} \geq 1$. From this point of view, the presented algorithm is able to distinguish between features of minimizers on three different length scales: the basic discretization length $\mathcal{O}(h)$, the thickness of the laminates $\mathcal{O}\left(h^{1-\beta}\right)$ and the thickness of the transition layers that connect the supports of different variants, $\mathcal{O}\left(h^{\beta_{i}}\right)$, with the latter being subject to refinement in the adaptive algorithm. In this scenario, $h>0$ is used as the principal discretization parameter that qualifies the accuracy of the method and determines the overall number of points constituting the meshes during the refinement process, i.e., $\mathcal{O}\left(h^{-3}\right)$ in three dimensions. In particular, it serves as the parameter to describe the initial quasiuniform triangulation $\mathcal{T}_{h}^{0} \equiv \mathcal{T}_{h}$. From this point of view of multiple scales, deformations $v_{h}^{i} \in \mathcal{A}_{h}^{i}$ (see definition below) are energetically favored that follow these requirements of different scalings.

The objective of the adaptive algorithm to be proposed below is to reduce the area where transition of martensitic variants occurs. Figure 1 shows the principal properties of the adaptive algorithm: the scope of it is to increase the flexibility in the minimization process and to avoid severe restrictions to the solution in this process that are caused by the underlying triangulation of the domain $\Omega$. - Let us start with the proposal of the adaptive energy functional.

Let the tuple $\left\{\mathcal{T}_{h}^{i}, \beta^{i}\right\}_{i=0}^{i_{F}}$ be given, and consider a modified energy for deformations $v_{h}^{i} \in \mathcal{A}_{h}^{i} \equiv \prod_{K \in \mathcal{T}_{h}^{i}} \mathcal{P}_{1}(K)$,

$$
\begin{aligned}
\mathcal{E}_{h, \beta}^{\beta_{i}}\left(v_{h}^{i}\right)= & \sum_{K \in \mathcal{T}_{h}^{i}} \int_{K} \phi\left(\nabla v_{h}^{i}(x)\right) \mathrm{d} \Omega+\alpha_{11}\left(\sum_{K \in \mathcal{T}_{h}^{i}} h^{2-\beta_{i}-\beta} \int_{\partial K}\left|\left[v_{h}^{i}\right](x)\right| \mathrm{d} \sigma\right)^{2} \\
& +\alpha_{12}\left(\sum_{K \in \mathcal{T}_{h}^{i}} h^{3-2 \beta_{i}-\beta} \int_{\partial K}\left|\left[v_{h}^{i}\right](x)\right|^{2} \mathrm{~d} \sigma\right)+\alpha_{2} \sum_{K \in \mathcal{T}_{h}^{i}} h^{2 \beta} \int_{\partial K \cap \partial \Omega}\left|v_{h}^{i}(x)-F_{\lambda} x\right|^{2} \mathrm{~d} \sigma
\end{aligned}
$$

for $0 \leq i \leq i_{F}$, and a choice $i_{F}=\mathcal{O}\left(\log _{2}\left(h^{-\beta / 2}\right)\right)$.

\section{Remark 3.1.}

1. The value for $i_{F}$ is a consequence of an elementary dimensionality argument for the applied refinement strategy and follows from the fact that we have $\mathcal{O}\left(h^{-3}\right)$ degrees of freedom (i.e., elements) to discretize 
the domain $\Omega \subset \mathbb{R}^{3}$. This numerical strategy favors minimizers exhibiting laminated microstructure, with the area supporting transitions of variants becoming smaller for increasing numbers of $i$. Since deformations are energetically preferred that have a number of $\mathcal{O}\left(h^{\beta-1}\right)$ layers which mark the transition between different variants in laminated microstructure, we are provided with not more than $\mathcal{O}\left(h^{-2-\beta}\right)$ degrees of freedom per each transition layer. From this consideration, the smallest triangles that come from a regular refinement process show a diameter of order $\mathcal{O}\left(h^{1+\beta / 2}\right)$.

2. If we carry out a corresponding investigation for a $2 \mathrm{D}$-version of the model (1), (8) for $\Omega \subset \mathbb{R}^{2}$ and for deformations $v \in \mathcal{B}$, where

$$
\mathcal{B}=\left\{v \in W^{1, \infty}\left(\Omega ; \mathbb{R}^{2}\right):\left.v(x)\right|_{\partial \Omega}=F_{\lambda} x\right\}
$$

we obtain a scaling of the area supporting transitions of variants that is different from the one in the 3D-case: the diameter of the smallest triangles that come from an admissible mesh refinement process can now be of size $\mathcal{O}\left(h^{1+\beta}\right)$. This implies that the value $i_{F}$ can now be chosen to be of order $\mathcal{O}\left(\log _{2}\left(h^{-\beta}\right)\right)$, and consequently $\beta_{i_{F}}=1+\beta$. Furthermore, as we see in the sequel, the value of $\beta$ also has to be adjusted to the spatial dimension $d \in\{2,3\}$ where minimizing deformations are studied.

3. The refinement process $\mathcal{S}$ can be accomplished in a regular way in that it keeps the quasiuniform character of the initial mesh $\mathcal{T}_{h}^{0}$ and avoids distorted meshes. For details on this construction of refined meshes, see $[1]$.

Then, the adaptive algorithm can be stated in the following way, for values $0 \leq i \leq i_{F}$, and with the initial quasiuniform triangulation $\mathcal{T}_{h}^{0} \equiv \mathcal{T}_{h}$ given from above:

1. For $v_{h}^{i} \in \mathcal{A}_{h}^{i}$ given, apply a regular refinement strategy

$$
\mathcal{S}_{i} \equiv \mathcal{S}_{i}\left(v_{h}^{i}\right): \mathcal{T}_{h}^{i} \rightarrow \mathcal{T}_{h}^{i+1}
$$

according to the following criterion: Refine by bisection adjacent elements $K \in \mathcal{T}_{h}^{i}$, that share a face $\mathcal{F} \subset \partial K$, where the jump of $v_{h}^{i}$ across $\mathcal{F}$ satisfies the following bound, for a given number $\alpha=\mathcal{O}(1)$,

$$
\max _{x \in \mathcal{F}}\left|\left[v_{h}^{i}\right](x)\right| \geq \alpha h^{\beta_{i}}
$$

2. Set $i:=i+1$ and go back to 1 . in case $i<i_{F}$. For $i=i_{F}$, go to the next step.

3. For $i=i_{F}$, compute a minimizer $u_{h}^{i_{F}} \in \mathcal{A}_{h}^{i_{F}}$ of

$$
\min _{v_{h}^{i_{F}} \in \mathcal{A}_{h}^{i_{F}}} \mathcal{E}_{h, \beta}^{\beta_{i_{F}}}\left(v_{h}^{i_{F}}\right), \quad \text { with } \quad \beta_{i_{F}}=1+\frac{\beta}{2} .
$$

Remark 3.2. Note that the second and third term with the leading factors $\alpha_{11}$ and $\alpha_{12}$ in (12) have a weight with respect to the basis $h$ and an exponent that couples the scaling of the laminate thickness with the one of the thickness of the transition area.

In order to assure a monotonous behavior of the algorithm and thus an optimal performance of the method, the refinement process $\mathcal{S}_{i}$ has to be properly chosen in the sense of the following definition.

Definition 1. Given a quasiuniform triangulation $\mathcal{T}_{h}^{0}:=\mathcal{T}$, we call a regular refinement strategy $\mathcal{S}:=$ $\left\{\mathcal{S}_{i}\right\}_{i=0}^{i_{F}-1}$, with $\mathcal{S}_{i}: \mathcal{T}_{h}^{i} \rightarrow \mathcal{T}_{h}^{i+1}$ admissible if there exists a sequence $\left\{\mathcal{T}_{h}^{i}, v_{h}^{i}\right\}_{i=0}^{i_{F}}$, and a constant $\tilde{C}=\tilde{C}\left(\alpha_{11}, \alpha_{12}\right)$ that is independent on the number $i$, for deformations $v_{h}^{i} \in \mathcal{A}_{h}^{i}$ satisfying

$$
\mathcal{E}_{h, \beta}^{\beta_{i}}\left(v_{h}^{i}\right) \leq \tilde{C} h^{2}, \quad \text { for } 0 \leq i \leq i_{F}
$$


The description of the simple laminated microstructure can be given in the following way: The orientation of it is determined by its normal vector $n \in \mathbb{R}^{3}$. In the following, we also make use of vectors $w \in \mathbb{R}^{3}$ along the laminates, which satisfy $w \cdot n=0$. Furthermore, we study the accuracy of representing the volume fractions $\lambda^{1}:=\lambda$ and $\lambda^{2}:=(1-\lambda)$. We refer to Section 4.4 for further details. - We are now in a position to present the main result of this work.

Theorem 3.1. Consider algorithm (13) through (15) as a discrete, adaptive scheme to solve problem (8, 9), with a sequence of triangulations $\mathcal{T}_{h}^{i}$ and values $1 \leq \beta_{i} \leq 1+\beta / 2$ given by the formula

$$
\beta_{i}=1+\log _{h}\left(2^{-i}\right)
$$

Then, there exists an admissible refinement strategy $\mathcal{S}$ of a given initial quasiuniform triangulation $\mathcal{T}_{h}^{0}=\mathcal{T}_{h}$, such that a minimizer $u_{h}^{i_{F}} \in \mathcal{A}_{h}^{i_{F}}$ of problem (15) satisfies the following approximation properties, for the optimal parameter choice $\beta=2 / 5$, and positive values $\alpha_{11}, \alpha_{12}, \alpha_{2}=\mathcal{O}(1)$,

(a) $\mathcal{E}_{h, 2 / 5}^{\beta_{i_{F}}}\left(u_{h}^{i_{F}}\right) \leq C h^{2}$,

(b) $\left\|u_{h}^{i_{F}}-F_{\lambda} x\right\|_{L^{2}(\Omega)} \leq C h^{3 / 10}$,

(c) $\left\|\left\{\nabla u_{h}^{i_{F}}-F_{\lambda}\right\} w\right\|_{L^{2}(\Omega)} \leq C h^{3 / 10}$,

(d) $\left|\frac{\mu\left(\omega_{\rho}^{\ell}\left(u_{h}^{i} F\right)\right)}{\mu(\omega)}-\lambda^{\ell}\right| \leq C h^{3 / 20}, \quad$ for $\ell \in\{1,2\}$,

for all subsets $\omega \subset \Omega$ and $h<\rho<1$ and a normalized vector $w \in \mathbb{R}^{3}$ that satisfies $w \cdot n=0$. The applied generic constant $C$ is only dependent on the parameters of the continuous problem (8) and the values $\alpha_{11}, \alpha_{12}, \alpha_{2}$ but does not dependent on the mesh size $h$ and $i_{F}$. In the cases $(d)$, the constant $C$ additionally depends on the choice of the positive number $\rho$.

\section{Remark 3.3.}

1. Note that the thickness of layers of the approximate simple laminated microstructure is now of magnitude $\mathcal{O}\left(h^{3 / 5}\right)$ - in contrast to the previous model (10), where minimizing deformations are energetically preferred that exhibit laminated microstructure of thickness $\mathcal{O}\left(h^{1 / 2}\right)$. The thickness of transition layers in between two bands of different variants is of magnitude $\mathcal{O}\left(h^{6 / 5}\right)$.

2. We emphasize the interplay between the adaptive construction of an admissible refinement strategy and the iterative adjustment of the energy functional with respect to the sequence $\left\{\beta_{i}\right\}_{i=0}^{i_{F}}$ in order to end up with the final form $\mathcal{E}_{h, \beta}^{\beta_{i_{F}}}(\cdot)$ : to some extent, the initial triangulation is made compatible to the laminated microstructure through the refinement strategy, whereas the modification of the energy functional enhances the continuity requirements on a computed deformation $v_{h}^{i}$ at each iteration step $0 \leq i \leq i_{F}$, only allowing for decreasing jumps across inter-element faces.

3. For computations, the admissible character of refined triangulations has to be checked at each iteration step; for instance, we can choose minimizing deformations $u_{h}^{i} \in \mathcal{A}_{h}^{i}$ of the energies

$$
\mathcal{E}_{h, 2 / 5}^{\beta_{i}}\left(v_{h}^{i}\right), \quad \forall v_{h}^{i} \in \mathcal{A}_{h}^{i}
$$

as indicators of a refinement strategy $\mathcal{S}$, but the admissible character of this strategy has to be verified.

4. The statements in Theorem 3.1 are given for the formulation of the energy functional (1) in 3D. According to Remark 3.1, the situation slightly changes, if we deal with this energy functional for deformations $v \in \mathcal{B}$ in two dimensions. In this case, the thickness of laminates is of order $\mathcal{O}\left(h^{2 / 3}\right)$ for the optimal parameter $\beta=1 / 3$, and the following results hold for positive $\alpha_{11}, \alpha_{12}, \alpha_{2}=\mathcal{O}(1)$,

(a) $\mathcal{E}_{h, 1 / 3}^{\beta_{i_{F}}}\left(u_{h}^{i_{F}}\right) \leq C h^{2}$,

(b) $\left\|u_{h}^{i_{F}}-F_{\lambda} x\right\|_{L^{2}(\Omega)} \leq C h^{1 / 3}$,

(c) $\left\|\left\{\nabla u_{h}^{i_{F}}-F_{\lambda}\right\} w\right\|_{L^{2}(\Omega)} \leq C h^{1 / 3}$, 
(d) $\left|\frac{\mu\left(\omega_{\rho}^{\ell}\left(u_{h}^{i} F\right)\right)}{\mu(\omega)}-\lambda^{\ell}\right| \leq C h^{1 / 6}, \quad$ for $\ell \in\{1,2\}$.

The verification of these statements follows from the analysis of the 3D case below and Remark 3.1.

The verification of Theorem 3.1 is given in Section 4. For its proof, we need to test a couple of auxiliary results. The general line of analyzing numerical schemes for rotationally invariant multi-well energy densities has first been developed in [24,26,27], and we also follow it in our proofs. Also, we benefit very much from the analysis of the discrete energy for discontinuous piecewise affine deformations (10) that is performed in [15] and refer to there in order to shorten the proofs.

\section{ANALYSis FOR THE DisCONTINUOUS Finite ELEMENT METHOD}

Given a number $i$, for $0 \leq i \leq i_{F}$, there are three terms in the energy functional (12) that compete with each other: the first is the bulk energy term that already comes from the continuous version $\mathcal{E}(\cdot)$. The two remaining terms weaken strict continuity constraints across adjacent elements that are satisfied in the continuous problem by penalizing (high) jumps. Finally, the last term in $\mathcal{E}_{h, \beta}^{\beta_{i}}(\cdot)$ allows for slight changes of the given (averaged) boundary data in a computed minimizer to improve the flexibility of the finite element method in order to resolve laminated microstructure on nonaligned grids.

\subsection{Discontinuous finite elements}

Given a triangulation $\mathcal{T}_{h}^{i}$, the Lagrange interpolation operator

$$
\mathcal{I}_{\mathcal{T}_{h}^{i}}: C\left(\bigcup_{K \in \mathcal{T}_{h}^{i}} K\right) \rightarrow \prod_{K \in \mathcal{T}_{h}^{i}} \operatorname{Aff}(K)
$$

with $\operatorname{Aff}(K)$ the set of affine-linear functions on each triangle $K \in \mathcal{T}_{h}^{i}$, is defined in a standard way as a point interpolate. From this, inverse inequalities are valid since they hold on each triangle, compare [9].

Lemma 4.1. Let $k$ and $l$ be two integers such that $0 \leq k \leq l \leq 2$. We have the following inverse inequalities for any $K \in \mathcal{T}_{h}^{i}$, and any $v_{h}^{i} \in \operatorname{Aff}(K)$,

1. $\left|v_{h}^{i}\right|_{l, K} \leq C h^{k-l}\left|v_{h}^{i}\right|_{k, K}, \quad \forall K \in \mathcal{T}_{h}^{i}$,

2. $\left|v_{h}^{i}\right|_{l, \infty, K} \leq C h^{k-l-3 / 2}\left|v_{h}^{i}\right|_{k, K}, \quad \forall K \in \mathcal{T}_{h}^{i}$.

\subsection{Properties of minimizers of the functional $\mathcal{E}_{h, \beta}^{\beta_{i_{F}}(\cdot)}$}

The next lemma shows that it is always possible to find deformations $u_{h}^{i} \in \prod_{K \in \mathcal{T}_{h}^{i}} \operatorname{Aff}(K)$ and an admissible refinement of $\mathcal{T}_{h} \equiv \mathcal{T}_{h}^{0}$ that satisfy $\mathcal{E}_{h, \beta}^{\beta_{i}}\left(u_{h}^{i}\right) \leq C h^{2}$, for $\beta \in[0,1]$, and $0 \leq i \leq i_{F}$.

Lemma 4.2. Given a quasiuniform triangulation $\mathcal{T}_{h}^{0} \equiv \mathcal{T}_{h}$. Then, there exists an admissible refinement strategy $\mathcal{S}$ and a minimizer $u_{h}^{i_{F}} \in \mathcal{A}_{h}^{i_{F}}$ satisfying the bound

$$
\mathcal{E}_{h, \beta}^{\beta_{i_{F}}}\left(u_{h}^{i_{F}}\right) \leq C h^{2}
$$

Proof. This result will be shown by an extended argument that constitutes the proof for the case $i=0$, as it is given in [15]. It will be presented in the following to show the existence of an admissible refinement strategy $\mathcal{S}$ giving triangulations $\left\{\mathcal{T}_{h}^{i}\right\}_{i=0}^{i_{F}}$ such that the above energy inequality is valid on $\mathcal{T}_{h}^{i_{F}}$. According to (2), we define a deformation $C(\Omega) \ni w: \Omega \rightarrow \mathbb{R}^{3}$,

$$
w(x)=\gamma h^{1-\beta} \tilde{w}\left(\frac{x}{\gamma h^{1-\beta}}\right) .
$$


Here, we set

$$
\tilde{w}(x)=F_{1} x+\left[\int_{0}^{x \cdot n} \xi(s) \mathrm{d} s\right] a,
$$

where $\xi(s): \mathbb{R} \rightarrow \mathbb{R}$ is a characteristic function with period 1 ,

$$
\xi(s)= \begin{cases}0 & \text { for all } 0 \leq s \leq \lambda, \\ 1 & \text { for all } \lambda<s<1\end{cases}
$$

and $\gamma=\mathcal{O}(1)$ is an arbitrary number. It is easy to see, that the following inequality holds true, with $\gamma$ incorporated into the constant $C$,

$$
\left|w(x)-F_{\lambda} x\right| \leq C h^{1-\beta}, \quad \forall x \in \Omega .
$$

We now have

$$
\nabla w(x)=F_{1}+\xi\left(\frac{x \cdot n}{\gamma h^{1-\beta}}\right) a \otimes n .
$$

In general, we are given a triangulation $\mathcal{T}_{h}^{0}=\left\{K_{l}^{0}\right\}_{l \in L^{0}}$ such that $w \neq \mathcal{I}_{\mathcal{T}_{h}^{0}}(w)$. Because of $w$ being piecewise affine there exists a refinement $\tilde{\mathcal{T}}_{h}^{0}=\left\{\tilde{K}_{l j}^{0}\right\}_{l \in L^{0}, j \in J_{l}^{0}}$ of $\mathcal{T}_{h}^{0}$, with

$$
\mathcal{T}_{h}^{0} \ni K_{l}^{0}=\bigcup_{j \in J_{l}^{0}} \tilde{K}_{l j}^{0}, \quad \tilde{K}_{l j}^{0} \in \tilde{\mathcal{T}}_{h}^{0}
$$

s.t. holds: $w=\mathcal{I}_{\tilde{\mathcal{T}}_{h}^{0}}(w)$.

In our notation, $\operatorname{card} J_{l}^{0}=1$ stands for no refinement, whereas card $J_{l}^{0}>1$ denotes a refinement of $K_{l}^{0} \in \mathcal{T}_{h}^{0}$. - We can now use the triangulation $\mathcal{T}_{h}^{0}$ to construct a deformation $v_{h}^{0} \in \mathcal{A}_{h}^{0}$ from $w$ presented in (17), such that $\mathcal{E}_{h, \beta}^{\beta_{0}}\left(v_{h}^{0}\right) \leq C h^{2}$. For this purpose,

$$
v_{h}^{0}(x)= \begin{cases}w(x), & \text { for all } K_{l}^{0} \in \mathcal{T}_{h}^{0}, \operatorname{such} \text { that } \operatorname{card} J_{l}^{0}=1, \\ \operatorname{Ext}_{K_{l}^{0}}(w)(x), & \text { for all } K_{l}^{0} \in \mathcal{T}_{h}^{0}, \operatorname{such} \text { that } \operatorname{card} J_{l}^{0}>1,\end{cases}
$$

where, for each $K_{l}^{0}$ such that $\operatorname{card} J_{l}^{0}>1$, we can define an extension operator

$$
\operatorname{Ext}_{K_{l}^{0}}: \prod_{\left\{\tilde{K}_{l j}^{0}\right\}_{j \in J_{l}^{0}}^{0}=K_{l}^{0} \in \mathcal{T}_{h}^{0}} \operatorname{Aff}\left(\tilde{K}_{l j}^{0}\right) \rightarrow \operatorname{Aff}\left(K_{l}^{0}\right),
$$

with $\operatorname{Ext}_{K_{l}^{0}}(w)(x)$ further defined to be a linear extension of $\left.w\right|_{\tilde{K}_{l j_{0}}^{0}}$ onto $\left\{\tilde{K}_{l j}^{0}\right\}_{j \in J_{l}^{0}}:=K_{l}^{0} \in \mathcal{T}_{h}^{0}$, satisfying

$$
\nabla \operatorname{Ext}_{K_{l}^{0}}(w)(x):=\left.\nabla w(x)\right|_{\tilde{K}_{l j_{0}}^{0}}, \quad \text { with } \mu\left(\tilde{K}_{l j_{0}}^{0}\right) \geq \mu\left(\tilde{K}_{l j}^{0}\right), \quad \forall j \in J_{l}^{0},
$$

for any $l \in L^{0}$. This gives a deformation candidate $v_{h}^{0}=\mathcal{I}_{\mathcal{T}_{h}^{0}}\left(v_{h}^{0}\right)$. It is now easy to compute the energy bound $\mathcal{E}_{h, \beta}^{\beta_{0}}\left(v_{h}^{0}\right) \leq C h^{2}$, thus verifying the statement of the Lemma for the choice $i=0$.

We now verify the existence of an admissible refinement strategy $\mathcal{S}$, with a sequence $\left\{\mathcal{T}_{h}^{i}, v_{h}^{i}\right\}_{i=0}^{i_{F}}$, for $v_{h}^{i} \in \mathcal{A}_{h}^{i}$. According to the definition, the deformation $v_{h}^{i-1} \in \mathcal{A}_{h}^{i-1}$ exhibits jumps close to the subsets of the domain $\Omega$ where a change of variants arises. Therefore, these elements are subject to a regular refinement according 
to $\mathcal{S}_{i-1}$, which gives a new mesh $\mathcal{T}_{h}^{i}$. For the following, we can make use of the previously introduced technical apparatus, by again choosing refined triangulations $\tilde{\mathcal{T}}_{h}^{i}=\left\{\tilde{K}_{l j}^{i}\right\}_{l \in L^{i}, j \in J^{i}}$ of the triangulation $\mathcal{T}_{h}^{i}=\left\{K_{l}^{i}\right\}_{l \in L^{i}}$, with

$$
\mathcal{T}_{h}^{i} \ni K_{l}^{i}=\bigcup_{j \in J_{l}^{i}} \tilde{K}_{l j}^{i}, \quad \tilde{K}_{l j}^{i} \in \tilde{\mathcal{T}}_{h}^{i},
$$

for all $0 \leq i \leq i_{F}$, where $w=\mathcal{I}_{\tilde{\mathcal{T}}_{h}}(w)$.

We can then define the following deformation $v_{h}^{i} \in \mathcal{A}_{h}^{i}$, for $i \geq 1$,

$$
v_{h}^{i}(x)= \begin{cases}v_{h}^{i-1}(x), & \text { for all } K_{l}^{i} \in \mathcal{T}_{h}^{i}, \text { such that } \operatorname{card} J_{l}^{i}=1, \\ \operatorname{Ext}_{K_{l}^{i}}(w)(x), & \text { for all } K_{l}^{i} \in \mathcal{T}_{h}^{i}, \text { such that card } J_{l}^{i}>1,\end{cases}
$$

with the extension operator $\operatorname{Ext}_{K_{l}^{i}}$ already defined before. — This provides us with candidates $v_{h}^{i} \in \mathcal{A}_{h}^{i}$, for $0 \leq i \leq i_{F}$, and it can be immediately seen that there holds $\mathcal{E}_{h, \beta}^{\beta_{i}}\left(v_{h}^{i}\right) \leq \tilde{C} h^{2}, 0 \leq i \leq i_{F}$. We thus presented an admissible refinement strategy $\mathcal{S}$, consisting of a sequence of tuples $\left\{v_{h}^{i}, \mathcal{T}_{h}^{i}\right\}_{i=0}^{i_{F}}$, with $v_{h}^{i} \in \mathcal{A}_{h}^{i}$.

The existence of a minimizer follows from standard arguments, see e.g. Lemma 2.2 in [15]. The upper energy bound for a minimizer $u_{h}^{i_{F}} \in \mathcal{A}_{h}^{i_{F}}$ on the final mesh $\mathcal{T}_{h}^{i_{F}}$ is immediate.

We can now benefit from this result. Let us recall that the continuity constraint given in the penalized energy functional $\mathcal{E}_{h, \beta}^{\beta_{i}}$ for the triangulation $\mathcal{T}_{h}^{i}$ is enforced for increasing numbers $i$. This is the consequence of the following theorem that plays a crucial role in the subsequent convergence analysis. Moreover, it shows that the adaptive energy functional (12) performs best for the choice $\beta=2 / 5$. - In the subsequent proof, we see the interplay of different features that arise in the algorithm, and that are scaled using the parameters $\beta$ and $\beta_{i}$.

Theorem 4.1. Suppose $\mathcal{T}_{h}^{i_{F}}$ to be constructed through an admissible refinement process. Then, a minimizer $u_{h}^{i_{F}} \in \mathcal{A}_{h}^{i_{F}}$ of the energy functional $\mathcal{E}_{h, \beta}^{\beta_{i}}(\cdot)$ that is given in (12) satisfies the following estimate,

$$
\left\|\sum_{K \in \mathcal{T}_{h}^{i_{F}}} \int_{K}\left\{\nabla u_{h}^{i_{F}}(x)-F_{\lambda}\right\} \mathrm{d} \Omega\right\| \leq C\left\{h^{3 \beta / 2}+h^{1-\beta}\right\}, \quad \text { for } \beta \in[0,1] .
$$

Proof. Take $\mathcal{A}_{h}^{i_{F}} \ni z_{h}^{i_{F}}(x)=u_{h}^{i_{F}}(x)-F_{\lambda} x$. Then, we have

$$
\begin{aligned}
\sum_{K \in \mathcal{T}_{h}^{i_{F}}} \int_{K} \nabla z_{h}^{i_{F}}(x) \mathrm{d} \Omega & =\sum_{K \in \mathcal{T}_{h}^{i_{F}}} \int_{\partial K} z_{h}^{i_{F}}(x) \otimes \nu \mathrm{d} \sigma \\
& =\sum_{\mathcal{F} \subset \partial K, K \in \mathcal{T}_{h}^{i_{F}}, \mathcal{F} \not \subset \partial \Omega} \int_{\mathcal{F}} z_{h}^{i_{F}}(x) \otimes \nu \mathrm{d} \sigma+\sum_{\mathcal{F} \subset \partial K, K \in \mathcal{T}_{h}^{i_{F}, \mathcal{F} \subset \partial \Omega}} \int_{\mathcal{F}} z_{h}^{i_{F}}(x) \otimes \nu \mathrm{d} \sigma \\
& :=I+I I .
\end{aligned}
$$


Owing to the fact that two neighboring elements share one face, with their related normal vectors changing their sign, we can continue with the first term as follows,

$$
\begin{aligned}
\||\|\mid\| & \leq\left\|\left.\sum_{\mathcal{F} \subset \partial K, K \in \mathcal{T}_{h}^{i_{F}}, \mathcal{F} \not \subset \partial \Omega} \int_{\mathcal{F}}\left(z_{h}^{i_{F},+}-z_{h}^{i_{F},-}\right)(x) \otimes \nu\right|_{K^{+}} \mathrm{d} \sigma \mid\right\| \\
& \leq \sum_{\mathcal{F} \subset \partial K, K \in \mathcal{T}_{h}^{i_{F}}, \mathcal{F} \not \subset \supset \Omega} \int_{\mathcal{F}}\left|\left[u_{h}^{i_{F}}\right](x)\right| \mathrm{d} \sigma \leq C h^{3 \beta / 2} .
\end{aligned}
$$

The last bound is a consequence of Lemma 4.2 and the evaluation $\beta_{i_{F}}=1+\beta / 2$ in the energy (12). Another application of Lemma 4.2 further leads to an upper bound for the term $I I$,

$$
\begin{aligned}
\|\| I \| & \leq \sum_{\mathcal{F} \subset \partial K, K \in \mathcal{T}_{h}^{i_{F}, \mathcal{F} \subset \partial \Omega}} \int_{\mathcal{F}}\left|z_{h}^{i_{F}}(x)\right| \mathrm{d} \sigma \leq C \sum_{\mathcal{F} \subset \partial K, K \in \mathcal{T}_{h}^{i_{F}}, \mathcal{F} \subset \partial \Omega} h\left(\int_{\mathcal{F}}\left|z_{h}^{i_{F}}(x)\right|^{2} \mathrm{~d} \sigma\right)^{1 / 2} \\
& \left.\leq C \sum_{\mathcal{F} \subset \partial K, K \in \mathcal{T}_{h}^{i_{F}, \mathcal{F} \subset \partial \Omega}} \int_{\mathcal{F}}\left|z_{h}^{i_{F}}(x)\right|^{2} \mathrm{~d} \sigma\right)^{1 / 2} \leq C h^{1-\beta} .
\end{aligned}
$$

This furnishes the proof of the theorem.

\section{Remark 4.1.}

1. If we consider a corresponding $2 D$-model, the following crucial bound is valid,

$$
\left\|\sum_{K \in \mathcal{T}_{h}^{i_{F}}} \int_{K}\left\{\nabla u_{h}^{i_{F}}(x)-F_{\lambda}\right\} \mathrm{d} \Omega\right\| \leq C\left\{h^{2 \beta}+h^{1-\beta}\right\}, \quad \text { for } \beta \in[0,1]
$$

This indicates $\beta=1 / 3$ to be the optimal value.

2. For conforming or classical nonconforming elements (see $[24,26]$ ), we have the sharper result

$$
\sum_{K \in \mathcal{T}_{h}} \int_{K}\left\{\nabla u_{h}(x)-F_{\lambda}\right\} \mathrm{d} \Omega=0
$$

3. A corresponding analysis has been given for problem (10) in [15], where the following error bound was proven, for $u_{h} \in \mathcal{A}_{h}$,

$$
\left\|\sum_{K \in \mathcal{T}_{h}} \int_{K}\left\{\nabla u_{h}(x)-F_{\lambda}\right\} \mathrm{d} \Omega\right\| \leq C\left\{h^{\beta}+h^{1-\beta}\right\}, \quad \text { for } \beta \in[0,1] .
$$

The following theorem quantifies the deviation of the computed minimizer $u_{h}^{i_{F}} \in \mathcal{A}_{h}^{i_{F}}$ from the minimizer $u \in \mathcal{A}$ in $L^{2}(\Omega)$. 
A. PROHL

Theorem 4.2. Suppose $\mathcal{T}_{h}^{i_{F}}$ to be obtained through an admissible refinement strategy $\mathcal{S}$ from $\mathcal{T}_{h}$. Then, a minimizer $u_{h}^{i_{F}} \in \mathcal{A}_{h}^{i_{F}}$ of $\mathcal{E}_{h, \beta}^{\beta_{i_{F}}(\cdot)}$ given in (12) satisfies the following estimate, for all normalized vectors $w \in \mathbb{R}^{3}$,

$$
\left(\sum_{K \in \mathcal{T}_{h}^{i_{F}}} \int_{K}\left|u_{h}^{i_{F}}(x)-F_{\lambda} x\right|^{2} \mathrm{~d} \Omega\right)^{1 / 2} \leq C\left\{\left(\sum_{K \in \mathcal{T}_{h}^{i_{F}}} \int_{K}\left|\left\{\nabla u_{h}^{i_{F}}-F_{\lambda}\right\} w\right|^{2} \mathrm{~d} \Omega\right)^{1 / 2}+h^{1-\beta}+h^{\beta}\right\} .
$$

Proof. We again make use of the abbreviated notation $z_{h}^{i_{F}}(x)=u_{h}^{i_{F}}(x)-F_{\lambda} x, x \in \Omega$. Using integration by parts, we find:

$$
\begin{aligned}
\int_{\Omega}\left|z_{h}^{i_{F}}(x)\right|^{2} \mathrm{~d} \Omega & =\sum_{K \in \mathcal{T}_{h}^{i_{F}}} \int_{\partial K}\left|z_{h}^{i_{F}}(x)\right|^{2}(w \cdot x)(w \cdot \nu) \mathrm{d} \sigma-\sum_{K \in \mathcal{T}_{h}^{i_{F}}} \int_{K}\left(\nabla\left|z_{h}^{i_{F}}(x)\right|^{2} \cdot w\right)(w \cdot x) \mathrm{d} \Omega \\
& :=I_{1}+I_{2},
\end{aligned}
$$

with an arbitrary vector $w \in \mathbb{R}^{3},|w|=1$. - The second term can be controlled as follows,

$$
\begin{aligned}
\left|I_{2}\right| & =\left|\sum_{K \in \mathcal{T}_{h}^{i_{F}}} \int_{K}\left(\nabla\left|z_{h}^{i_{F}}(x)\right|^{2} \cdot w\right)(w \cdot x) \mathrm{d} \Omega\right| \\
& \leq C \max _{x \in \bar{\Omega}}|w \cdot x|\left(\sum_{K \in \mathcal{T}_{h}^{i_{F}}} \int_{K}\left|\nabla z_{h}^{i_{F}}(x) w\right|^{2} \mathrm{~d} \Omega\right)^{1 / 2}\left(\int_{\Omega}\left|z_{h}^{i_{F}}(x)\right|^{2} \mathrm{~d} \Omega\right)^{1 / 2} \\
& \leq \frac{1}{4} \int_{\Omega}\left|z_{h}^{i_{F}}(x)\right|^{2} \mathrm{~d} \Omega+C \sum_{K \in \mathcal{T}_{h}^{i_{F}}} \int_{K}\left|\nabla z_{h}^{i_{F}}(x) w\right|^{2} \mathrm{~d} \Omega .
\end{aligned}
$$

In order to handle the term $I_{1}$, we distinguish between the edges in the interior of the domain and those on the boundary $\partial \Omega$,

$$
\begin{aligned}
I_{1} & =\sum_{\mathcal{F} \subset \partial K, K \in \mathcal{T}_{h}^{i_{F}}, \mathcal{F} \subset \partial \Omega} \int_{\mathcal{F}}\left|z_{h}^{i_{F}}(x)\right|^{2}(w \cdot x)(w \cdot \nu) \mathrm{d} \sigma+\sum_{\mathcal{F} \subset \partial K, K \in \mathcal{T}_{h}^{i_{F}, \mathcal{F} \not \subset \partial \Omega}} \int_{\mathcal{F}}\left|z_{h}^{i_{F}}(x)\right|^{2}(w \cdot x)(w \cdot \nu) \mathrm{d} \sigma \\
& :=I_{11}+I_{12} .
\end{aligned}
$$

Because of the existence of a minimizer $u_{h}^{i_{F}} \in \mathcal{A}_{h}^{i_{F}}$ satisfying the energy bound $\mathcal{E}_{h, \beta}^{\beta_{i_{F}}}\left(u_{h}^{i_{F}}\right) \leq C h^{2}$ according to Lemma 4.2, we immediately obtain

$$
\left|I_{11}\right| \leq C \sum_{\mathcal{F} \subset \partial K, K \in \mathcal{T}_{h}^{i_{F}}, \partial K \subset \partial \Omega} \int_{\mathcal{F}}\left|z_{h}^{i_{F}}(x)\right|^{2} \mathrm{~d} \sigma \leq C h^{2(1-\beta)} .
$$


In order to bound $I_{12}$, we shall distinguish along each face between $\nu^{+}$and $\nu^{-}$, depending on the direction of the outer normal of adjacent triangles $K^{+}, K^{-} \in \mathcal{T}_{h}^{i_{F}}$, for $\overline{K^{+}} \cap \overline{K^{-}} \neq \emptyset$,

$$
\begin{aligned}
I_{12} & =\sum_{\mathcal{F} \subset \partial K, \mathcal{F} \not \subset \partial \Omega} \int_{\mathcal{F}}\left\{\left|z_{h}^{i_{F},+}(x)\right|^{2}(w \cdot x)\left(w \cdot \nu^{+}\right)+\left|z_{h}^{i_{F},-}(x)\right|^{2}(w \cdot x)\left(w \cdot \nu^{-}\right)\right\} \mathrm{d} \sigma \\
& =\sum_{\mathcal{F} \subset \partial K, \mathcal{F} \not \subset \partial \Omega} \int_{\mathcal{F}}\left\{\left|z_{h}^{i_{F},+}(x)\right|^{2}-\left|z_{h}^{i_{F},-}(x)\right|^{2}\right\}(w \cdot x)\left(w \cdot \nu^{+}\right) \mathrm{d} \sigma \\
& =\sum_{\mathcal{F} \subset \partial K, \mathcal{F} \not \subset \partial \Omega} \int_{\mathcal{F}}\left(\left\{z_{h}^{i_{F},+}-z_{h}^{i_{F},-}\right\}(x) \cdot\left\{z_{h}^{i_{F},+}+z_{h}^{i_{F},-}\right\}(x)\right)(w \cdot x)\left(w \cdot \nu^{+}\right) \mathrm{d} \sigma \\
& =\sum_{\mathcal{F} \subset \partial K, \mathcal{F} \not \subset \partial \Omega} \int_{\mathcal{F}}\left(\left[u_{h}^{i_{F}}\right](x) \cdot\left\{z_{h}^{i_{F},+}+z_{h}^{i_{F},-}\right\}(x)\right)(w \cdot x)\left(w \cdot \nu^{+}\right) \mathrm{d} \sigma \\
& \leq C \sum_{\mathcal{F} \subset \partial K, \mathcal{F} \not \subset \partial \Omega} \int_{\mathcal{F}}\left|\left[u_{h}^{i_{F}}\right](x)\right|\left\{\left|z_{h}^{i_{F},+}(x)\right|+\left|z_{h}^{i_{F},-}(x)\right|\right\} \mathrm{d} \sigma \\
& \leq C \sum_{\mathcal{F} \subset \partial K, \mathcal{F} \not \subset \partial \Omega}\left(\int_{\mathcal{F}}\left|\left[u_{h}^{i_{F}}\right](x)\right|^{2} \mathrm{~d} \sigma\right)^{1 / 2}\left(\int_{\mathcal{F}}\left\{\left|z_{h}^{i_{F},+}(x)\right|+\left|z_{h}^{i_{F},-}(x)\right|\right\}^{2} \mathrm{~d} \sigma\right)^{1 / 2} .
\end{aligned}
$$

Because of Lemma 4.1, we can continue,

$$
\begin{aligned}
& \leq C \sum_{\mathcal{F} \subset \partial K, \mathcal{F} \not \subset \partial \Omega}\left(\int_{\mathcal{F}}\left|\left[u_{h}^{i_{F}}\right](x)\right|^{2} \mathrm{~d} \sigma\right)^{1 / 2} h_{K}^{-1 / 2}\left(\int_{K}\left|z_{h}^{i_{F}}(x)\right|^{2} \mathrm{~d} \Omega\right)^{1 / 2} \\
& \leq C \sum_{\mathcal{F} \subset \partial K, \mathcal{F} \not \subset \partial \Omega} \int_{\mathcal{F}} \frac{1}{h_{K}}\left|\left[u_{h}^{i_{F}}\right](x)\right|^{2} \mathrm{~d} \sigma+\frac{1}{4} \int_{\Omega}\left|z_{h}^{i_{F}}(x)\right|^{2} \mathrm{~d} \Omega .
\end{aligned}
$$

Because of the result

$$
\sum_{K \in \mathcal{T}_{h}^{i_{F}}} \int_{\partial K}\left|\left[u_{h}^{i_{F}}\right](x)\right|^{2} \mathrm{~d} \sigma \leq C h^{1+2 \beta},
$$

which is an immediate consequence of Lemma 4.2, we can now insert (28) through (33) in (27) and the proof is finished.

The following theorem gives a bound for errors on interior edges in terms of the error on elements. For this purpose, we will introduce subdomains $\omega_{h} \subset \bar{\Omega}$ being "pseudo-parallelepipeds". By this, we mean a perturbed parallelepiped, with the surface assembled from piecewise affine curves that are pointwise $\mathcal{O}(h)$ perturbations of the parallelepiped $\omega \supset \omega_{h}$.

Theorem 4.3. Assume $\left\{\mathcal{T}_{h}^{i}\right\}_{i=1}^{i_{F}}$ to be a sequence of admissibly refined triangulations. Then, there exists a constant $C=C(\omega)>0$ such that for any pseudo-parallelepiped $\omega_{h}=\left\{K_{s}\right\}_{s \in L} \subset \bar{\Omega}$ which is a union of elements $K_{s} \in \mathcal{T}_{h}^{i_{F}}=\left\{K_{s}\right\}_{s \in I}$, with $I \supset L$, the following is valid,

$$
\begin{aligned}
\left(\sum_{\mathcal{F} \subset \partial K, \mathcal{F} \cap \partial \omega_{h} \neq \emptyset, K \in \mathcal{T}_{h}^{i_{F}}, K \subset \omega_{h}} \int_{\mathcal{F}}\left|u_{h}^{i_{F}, \bullet}(x)-F_{\lambda} x\right|^{2} \mathrm{~d} \sigma\right)^{1 / 2} \leq \frac{C}{\Lambda^{1 / 2}(\omega)}\left(\int_{\omega_{h}}\left|u_{h}^{i_{F}}(x)-F_{\lambda} x\right|^{2} \mathrm{~d} \Omega\right)^{1 / 2}+C h^{\beta} \\
+C\left(\int_{\omega_{h}}\left|u_{h}^{i_{F}}(x)-F_{\lambda} x\right|^{2} \mathrm{~d} \Omega\right)^{1 / 4}\left(\sum_{K \subset \omega_{h}} \int_{K}\left|\| \nabla u_{h}^{i_{F}}(x)-F_{\lambda}\right|||^{2} \mathrm{~d} \Omega\right)^{1 / 4},
\end{aligned}
$$


where $\Lambda(\omega)$ is the length of the shortest edge of the corresponding parallelepiped $\omega$ and where $\left.u_{h}^{i_{F}, \bullet}\right|_{\partial K \cap \partial \omega_{h} \neq \emptyset}$ is the trace of $\left.u_{h}^{i_{F}}\right|_{K \subset \omega_{h}}$ on $\partial K \cap \partial \omega_{h}$.

Remark 4.2. We omit the proof of this theorem and refer to Theorem 2.3 and its proof in [15]. Note that the improved second term on the right hand side is a consequence of the application of inequality (33).

\subsection{Approximation of limiting macroscopic deformations}

The results that are presented in this section for the adaptive energy (12) are improved versions of corresponding theorems which are verified in [15] for the energy (10). The improved orders on the right hand side of the subsequent estimates are based on the results that were derived in the previous section. - Since the proofs of the subsequent statements can immediately be copied from corresponding ones in [15] by taking benefit from the Theorems 4.1, 4.2 and 4.3, we will omit them here and just present the results.

Lemma 4.3. Suppose $\left\{\mathcal{T}_{h}^{i}\right\}_{i=0}^{i_{F}}$ to be a sequence of admissibly refined triangulations. Then there holds,

$$
\sum_{K \in \mathcal{T}_{h}^{i_{F}}} \int_{K}\left\|\nabla v_{h}^{i_{F}}(x)-\pi\left(\nabla v_{h}^{i_{F}}(x)\right)\right\| \|^{2} \mathrm{~d} \Omega \leq C \mathcal{E}_{h, \beta}^{\beta_{i_{F}}}\left(v_{h}^{i_{F}}\right), \quad \forall v_{h}^{i_{F}} \in \mathcal{A}_{h}^{i_{F}} .
$$

The next two statements quantify the accuracy of the computed minimizer along the laminates.

Lemma 4.4. Suppose $\left\{\mathcal{T}_{h}^{i}\right\}_{i=0}^{i_{F}}$ to be a sequence of admissibly refined triangulations. Then, for any $w \in \mathbb{R}^{3}$ satisfying $w \cdot n=0$, there exists a constant $C>0$ such that the following bound holds for a minimizer $u_{h}^{i_{F}} \in \mathcal{A}_{h}^{i_{F}}$,

$$
\left(\sum_{K \in \mathcal{T}_{h}^{i_{F}}} \int_{K}\left|\left\{\pi\left(\nabla u_{h}^{i_{F}}(x)\right)-F_{\lambda}\right\} w\right|^{2} \mathrm{~d} \Omega\right)^{1 / 2} \leq C\left\{\left(\mathcal{E}_{h, \beta}^{\beta_{i_{F}}}\left(u_{h}^{i_{F}}\right)\right)^{1 / 4}+h^{3 \beta / 4}+h^{(1-\beta) / 2}\right\}
$$

with $u_{h}^{i_{F}} \in \mathcal{A}_{h}^{i_{F}}$ being a minimizer of problem (11).

Theorem 4.4. Suppose $\left\{\mathcal{T}_{h}^{i}\right\}_{i=0}^{i_{F}}$ to be a sequence of admissibly refined triangulations. For any $w \in \mathbb{R}^{3}$ satisfying $w \cdot n=0$, there exists a constant $C>0$ such that

$$
\left(\sum_{K \in \mathcal{T}_{h}^{i_{F}}} \int_{K}\left|\left\{\nabla u_{h}^{i_{F}}(x)-F_{\lambda}\right\} w\right|^{2} \mathrm{~d} \Omega\right)^{1 / 2} \leq C\left\{\left(\mathcal{E}_{h, \beta}^{\beta_{i_{F}}}\left(u_{h}^{i_{F}}\right)\right)^{1 / 4}+h^{3 \beta / 4}+h^{(1-\beta) / 2}\right\} .
$$

The next theorem states in what way the gradients of energy-minimizing sequences of deformations converge weakly to $F_{\lambda}$ with respect to the basic discretization parameter $h$.

Theorem 4.5. Suppose $\left\{\mathcal{T}_{h}^{i}\right\}_{i=0}^{i_{F}}$ to be a sequence of admissibly refined triangulations. For any parallelepiped $\omega \subset \bar{\Omega}$, there exists a constant $C=C(\omega)>0$, such that the following bound holds for a minimizer $u_{h}^{i_{F}} \in \mathcal{A}_{h}^{i_{F}}$,

$$
\begin{aligned}
\left\|\sum_{K \in \mathcal{T}_{h}^{i_{F}}, K \cap \omega_{h} \neq \emptyset} \int_{K}\left\{\nabla u_{h}^{i_{F}}(x)-F_{\lambda}\right\} \mathrm{d} \Omega\right\| \leq & C\left\{h+h^{1 / 2}\left(\mathcal{E}_{h, \beta}^{\beta_{i_{F}}}\left(u_{h}^{i_{F}}\right)\right)^{1 / 2}+h^{3 \beta / 2}\right. \\
& \left.+\left(\mathcal{E}_{h, \beta}^{\beta_{i_{F}}}\left(u_{h}^{i_{F}}\right)\right)^{1 / 8}+h^{(1-\beta) / 4}+h^{3 \beta / 8}\right\} .
\end{aligned}
$$

\subsection{Approximation of simply laminated microstructure}

Again, the verification of the results presented here makes use of the sharp inequalities presented in the last section, and the proofs of corresponding statements given in [15] can be immediately applied. From this reason, 
we again omit the presentation of the corresponding proofs, and refer the interested reader to [15]. — For the formulation of the results, let us introduce some additional notation.

For any subset $\omega \subset \Omega, \rho>0$ and $v_{h}^{i} \in \mathcal{A}_{h}^{i}$, with $0 \leq i \leq i_{F}$, we introduce the sets

$$
\omega_{\rho}^{\ell}\left(v_{h}^{i}\right)=\bigcup_{K \in \mathcal{T}_{h}}\left\{x \in \omega \cap K: \Pi\left(\nabla v_{h}^{i}(x)\right)=F_{\ell}, \text { and }\left\|\nabla v_{h}^{i}(x)-F_{\ell}\right\|<\rho\right\},
$$

for $\ell \in\{1,2\}$. - Here, we make use of the operator $\Pi: \mathbb{R}^{3 \times 3} \rightarrow\left\{F_{1}, F_{2}\right\}$ that is related to the operator $\pi$ in the following way,

$$
\pi(F)=\Theta(F) \Pi(F), \quad \text { with } \quad \Theta: \mathbb{R}^{3 \times 3} \rightarrow S O(3), \quad \forall F \in \mathbb{R}^{3 \times 3} .
$$

Then we have the following result.

Theorem 4.6. Suppose $\left\{\mathcal{T}_{h}^{i}\right\}_{i=0}^{i_{F}}$ to be a sequence of admissibly refined triangulations. There exists a constant $C>0$ such that a minimizer $u_{h}^{i_{F}} \in \mathcal{A}_{h}^{i_{F}}$ satisfies the following bound,

$$
\left(\sum_{K \in \mathcal{T}_{h}^{i} F} \int_{K}\|\| u_{h}^{i_{F}}(x)-\Pi\left(\nabla u_{h}^{i_{F}}(x)\right)\|\|^{2} \mathrm{~d} \Omega\right)^{1 / 2} \leq C\left\{\left(\mathcal{E}_{h, \beta}^{\beta_{i_{F}}}\left(u_{h}^{i_{F}}\right)\right)^{1 / 4}+h^{3 \beta / 4}+h^{(1-\beta) / 2}\right\} .
$$

Finally, the following theorem quantifies the ability of the adaptive method $(14,15)$ to approximate the laminated microstructure.

Theorem 4.7. Suppose $\left\{\mathcal{T}_{h}^{i}\right\}_{i=0}^{i_{F}}$ to be a sequence of admissibly refined triangulations. For any rectangular parallelepiped $\omega \subset \Omega$, and any $\rho>0$, there exists a constant $C=C(\omega, \rho)>0$, such that a minimizer $u_{h}^{i_{F}} \in \mathcal{A}_{h}^{i_{F}}$ enjoys the following bound, for $\ell \in\{1,2\}$,

$$
\left|\frac{\mu\left(\omega_{\rho}^{\ell}\left(u_{h}^{i_{F}}\right)\right)}{\mu(\omega)}-\lambda^{\ell}\right| \leq C\left\{\left(\mathcal{E}_{h, \beta}^{\beta_{i_{F}}}\left(u_{h}^{i_{F}}\right)\right)^{1 / 8}+h^{3 \beta / 8}+h^{(1-\beta) / 4}\right\}
$$

This investigation furnishes Theorem 3.1.

The research was conducted during my stay at the IMA, University of Minnesota, and has been supported by a DFGscholarship. I thank the IMA for the hospitality. In particular, I am grateful to M. Luskin for introducing me to the topic of microstructure and to M. Gobbert for many stimulating discussions.

\section{REFERENCES}

[1] R.E. Bank, PLTMG: A Software Package for Solving Elliptic Partial Differential Equations. User's Guide 6.0. SIAM Philadelphia (1990).

[2] J. Ball and R. James, Fine phase mixtures as minimizers of energy. Arch. Rational Mech. Anal. 100 (1987) 13-52.

[3] J. Ball and R. James, Proposed experimental tests of a theory of fine microstructure and the two-well problem. Philos. Trans. Roy. Soc. London Ser. A 338 (1992) 389-450.

[4] C. Carstensen and P. Plechac, Numerical solution of the scalar double-well problem allowing microstructure. Math. Comp. 66 (1997) 997-1026.

[5] M. Chipot, Numerical analysis of oscillations in nonconvex problems. Numer. Math. 59 (1991) 747-767.

[6] M. Chipot and C. Collins, Numerical approximations in variational problems with potential wells. SIAM J. Numer. Anal. 29 (1992) 1002-1019.

[7] M. Chipot, C. Collins and D. Kinderlehrer, Numerical analysis of oscillations in multiple well problems. Numer. Math. 70 (1995) 259-282.

[8] C. Collins, Computation and Analysis of Twinning in Crystalline Solids, Ph.D. thesis, University of Minnesota, USA (1990).

[9] P. Ciarlet, The finite element method for elliptic problems. North-Holland, Amsterdam (1978). 
[10] C. Collins, D. Kinderlehrer and M. Luskin, Numerical approximation of the solution of a variational problem with a double well potential. SIAM J. Numer. Anal. 28 (1991) 321-332.

[11] C. Collins and M. Luskin, Optimal order estimates for the finite element approximation of the solution of a nonconvex variational problem. Math. Comp. 57 (1991) 621-637.

[12] J. Ericksen, Constitutive theory for some constrained elastic crystals. J. Solids and Structures 22 (1986) 951-964.

[13] J. Ericksen, Some constrained elastic crystals, in Material Instabilities in Continuum Mechanics and Related Problems, J. Ball Ed., Oxford University Press, Oxford (1987) 119-137.

[14] J. Ericksen, Twinning of crystals I, in Metastability and Incompletely Posed Problems, S. Antman, J. Ericksen, D. Kinderlehrer and I. Müller Eds., Springer-Verlag, New York (1987) 77-96; IMA Volumes in Mathematics and Its Applications, Vol 3.

[15] M. Gobbert and A. Prohl, A discontinuous finite element method for solving a multi-well problem. Technical Report 1539, IMA (1998) and SIAM J. Numer. Anal. (to be published).

[16] M. Gobbert and A. Prohl, A survey of classical and new finite element methods for the computation of crystalline microstructure. Technical Report 1576, IMA (1998).

[17] P. Gremaud, Numerical analysis of a nonconvex variational problem related to solid-solid phase transitions. SIAM J. Numer. Anal. 31 (1994) 111-127.

[18] P. Kloucek, B. Li and M. Luskin, Analysis of a class of nonconforming finite elements for crystalline microstructure. Math. Comp. 67 (1996) 1111-1125.

[19] P. Kloucek and M. Luskin, The computation of the dynamics of martensitic microstructure. Contin. Mech. Thermodyn. 6 (1994) 209-240.

[20] M. Kruzik, Numerical approach to double well problems. SIAM J. Numer. Anal. 35 (1998) 1833-1849.

[21] M. Kruzik, Oscillations, Concentrations and Microstructure Modeling, Ph.D. thesis, Charles University, Prague, Czech Republic (1996).

[22] B. Li, Analysis and Computation of Martensitic Microstructure, Ph.D. thesis, University of Minnesota, USA (1996).

[23] B. Li and M. Luskin, Finite element analysis of microstructure for the cubic to tetragonal transformation. SIAM J. Numer. Anal. 35 (1998) 376-392.

[24] B. Li and M. Luskin, Nonconforming Finite element approximation of crystalline microstructure. Math. Comp. 67 (1998) 917-946.

[25] B. Li and M. Luskin, Approximation of a martensitic laminate with varying volume fractions. RAIRO Modél. Math. Anal. Numér. 33 (1999) 67-87.

[26] M. Luskin, Approximation of a laminated microstructure for a rotationally invariant, double well energy density. Numer. Math. 75 (1997) 205-221.

[27] M. Luskin, On the computation of crystalline microstructure. Acta Numer. 5 (1996) 191-257. 